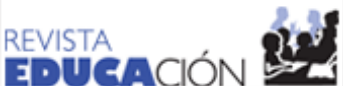

Revista Educación

ISSN: 0379-7082

ISSN: 2215-2644

revedu@gmail.com

Universidad de Costa Rica

Costa Rica

\section{Competencias digitales en estudiantes de educación secundaria de una provincia del centro del Perú}

Orosco Fabian, Jhon Richard; Gómez Galindo, Wilfredo; Pomasunco Huaytalla, Rocío; Salgado Samaniego, Edwin; Álvarez Casabona, Rodrigo César

Competencias digitales en estudiantes de educación secundaria de una provincia del centro del Perú

Revista Educación, vol. 45, núm. 1, 2021

Universidad de Costa Rica, Costa Rica

Disponible en: http://www.redalyc.org/articulo.oa?id=44064134008

DOI: https://doi.org/10.15517/revedu.v45i1.41296

\section{(c) (1) $\Theta(9$}

Esta obra está bajo una Licencia Creative Commons Atribución-NoComercial-SinDerivar 3.0 Internacional. 


\title{
Competencias digitales en estudiantes de educación secundaria de una provincia del centro del Perú
}

\author{
Digital Skills among High School Students in a Central Peruvian Province
}

Jhon Richard Orosco Fabian

Universidad Nacional del Centro del Perú, Perú

DOI: https://doi.org/10.15517/revedu.v45i1.41296

jorosco@uncp.edu.pe

iD http://orcid.org/0000-0001-9035-706X

Wilfredo Gómez Galindo

Universidad Nacional del Centro del Perú, Perú

wgomez@uncp.edu.pe

(iD) http://orcid.org/0000-0002-9946-0538

Rocio Pomasunco Huaytalla

Universidad Nacional del Centro del Perú, Perú

rpomasunco@uncp.edu.pe

(iD) http://orcid.org/0000-0002-8656-1479

Edwin Salgado Samaniego

Universidad Nacional del Centro del Perú, Perú

esalgado@uncp.edu.pe

(iD http://orcid.org/0000-0002-4473-479X

Rodrigo César Álvarez Casabona

Universidad Nacional del Centro del Perú, Tarma, Perú

rcalvarezcasabona@gmail.com

(D) http://orcid.org/0000-0002-7558-0551

Recepción: 16 Abril 2020

Aprobación: 21 Agosto 2020

\section{RESUMEN:}

El estudio analiza el logro de competencias digitales de la población estudiantil de educación secundaria, según sexo y grado. La investigación es de enfoque cuantitativo, de diseño transversal descriptivo. La muestra estuvo compuesta por 665 estudiantes de las instituciones educativas públicas de una provincia de la región central del Perú. Se recogió información a través de un instrumento elaborado con base en la propuesta del Instituto Nacional de Tecnologías Educativas y de Formación del Profesorado (2017) sobre competencias digitales agrupadas en cinco áreas (información y alfabetización informacional, comunicación y colaboración, creación de contenidos digitales, seguridad y resolución de problemas). Los resultados muestran que el estudiantado presenta un nivel de logro esperado en áreas de las competencias digitales de: información y alfabetización informacional (70.1\%), seguridad (61.8\%), creación de contenidos digitales (48.4\%), asimismo, en comunicación y colaboración (47.4\%). En el área de la competencia resolución de problemas es de $54.3 \%$, predomina un nivel de logro en proceso. Se concluye que en más del $50 \%$ de estudiantes de educación secundaria prevalecen las competencias digitales en un nivel de logro esperado, además que existe diferencia significativa en cuanto a las variables sexo $(\mathrm{p}=.012)$ y grado de educación $(\mathrm{p}=<.00)$, por tanto, se recomienda al personal docente integrar estas competencias en el proceso de aprendizaje para su fortalecimiento, además la implementación de infraestructura tecnológica por parte de las autoridades educativas y políticas.

Palabras clave: Competencias digitales, Alfabetización digital, Tecnologías de la Información y Comunicación, TIC, Estudiante de secundaria, Educación.

\section{Abstract:}


The study analyzes digital skills among a sample of the high-school student population based on gender and grade, using a quantitative approach with a descriptive crosscutting design. The sample included 665 students from public schools in a Central Peru province. Information was collected through a questionnaire that we developed based on the National Institute of Educational Technologies and Teacher Training (2017) recommendations regarding digital skills. The survey was grouped into five areas (Information and Information Literacy, Communication and Collaboration, Creation of Digital Content, Security and Problem Solving). According to the results, student body digital skill levels had an expected level of achievement with the following results: Information and Information Literacy (70.1\%), Security (61.8\%), Digital Content Creation (48.4\%), and Communication and Collaboration (47.4\%). Digital Skills Problem Resolutions Processes predominate at 54.3\% It can be concluded that digital skills levels were prevailing among more than $50 \%$ of high-school were as expected. The terms of the gender variables were $(p=.012)$ and school grade $(p=<.00)$. It is recommended that teachers incorporate these skills to strengthen the learning process and that educational and political authorities implement technology infrastructure.

KEYWORDS: Digital Skills, Digital Literacy, Information and communications technology, ICT, High-School Students, Education.

\section{INTRODUCCIÓN}

El desarrollo de la informática a través de sus diferentes recursos genera avances cada vez más vertiginosos de las Tecnologías de la Información y Comunicación (TIC), lo cual permite nuevas formas de almacenar, procesar y transmitir información, cuyas principales características son su portabilidad, inmediatez y globalidad (Pérez, 2017). Este fenómeno viene cambiando las tradicionales formas de desarrollar las actividades (Calderón, 2019).

La sociedad actual denominada sociedad de la información o conocimiento, es caracterizada por el uso creciente e incesante de las TIC, las cuales afectan las actividades humanas y las relaciones entre las personas; en el ámbito educativo, obliga a asumir nuevos retos; según Área (2009), citado por el Programa Aprende de la Junta de Castilla y León [PAJCL](2011), los nuevos desafíos educativos ante el incesante desarrollo de las tecnologías son analfabetismo tecnológico, saturación de la información, inadaptación a la rapidez de los cambios, dificultades para entender los nuevos formatos de texto, desajuste de los sistemas de formación, nuevas exigencias formativas ocupacionales y, por ende, nuevas competencias que exigen el contexto actual; por ejemplo, Cruz, Pozo, Aushay y Arias (2019) encontraron que el cuerpo docente no comprende eficazmente la utilidad del uso de las TIC; Fernández (2018) menciona que se exige a la educación que desarrolle nuevas competencias y habilidades para que el estudiantado se desenvuelva en un contexto de cambios constantes y aprendizaje continuo, y Levano, Sánchez, Guillén, Tello, Herrera y Collantes (2019) identificaron que la mayoría de adultos muestran, casi o completa nulidad en el uso de recursos tecnológicos, por lo que este contexto conllevará a diferenciar entre los que adquieren ciertas competencias digitales y aquellos que no. Al considerar lo mencionado, el estudiantado debería recibir formación para emplear todo un conjunto de tecnologías y competencias, incluida la capacidad de reflexionar sobre el mundo digital y sobre su experiencia en él, tanto en su vida como en su aprendizaje (Biasini, 2018).

Cada vez más, las TIC influyen directamente en diversos aspectos de la formación del estudiantado, porque con estos recursos digitales, el aprendizaje ya no es exclusivamente sincrónico, sino que las instituciones educativas progresivamente van ofreciendo y desarrollando sus servicios de manera asincrónica o mixta.

El contexto peruano no es ajeno a esta realidad, sobre todo en el ámbito educativo, en donde debido a las exigencias del contexto en general, las instituciones de formación, sean de educación básica, superior o superior universitaria, están obligadas a implementar e integrar los recursos digitales en sus procesos formativos, los cuales deben generar el desarrollo de competencias digitales en el estudiantado (Mateus y Suárez, 2017).

En gran parte de estudios (Bielva, Martínez, Herrera y Rodríguez, 2015; Cruz, 2019; Fernández y Manzano, 2018; Matamala, 2015; Muñoz y Nicaragua, 2014; Nieto, 2018; Sandí y Sanz, 2020) las competencias digitales han sido evaluadas parcialmente, en otros contextos y niveles educativos o alineadas 
a otros factores, en el contexto peruano no existen estudios al respecto, con instrumentos actualizados y contextualizados que engloben competencias digitales propuestas por reconocidas organizaciones que fomentan el desarrollo de tecnologías educativas como el Instituto Nacional de Tecnologías Educativas y de Formación del Profesorado [Intef], 2017), ello motivó elaborar el estudio en el contexto de la región central del Perú, con el propósito de analizar el alcance de diferentes competencias digitales vinculadas con la información y alfabetización informacional, comunicación y colaboración, creación de contenidos digitales, seguridad y resolución de problemas por parte de la población estudiantil a nivel de educación secundaria según sexo y grado de estudios.

El documento se encuentra organizado de la siguiente manera, el apartado 1 aborda la introducción del estudio, donde se describe la problemática y el propósito; el apartado 2 corresponde a los estudios previos a nivel mundial y latinoamericano; en el apartado 3 se desarrolla los referentes conceptuales respecto a competencias digitales; en el apartado 4 se considera los procedimientos metodológicos como el tamaño de muestra, desarrollo del instrumento de recojo de datos y el procedimiento de recojo y análisis de datos; en el apartado 5 se considera los resultados de las 21 competencias digitales estudiadas, así como el análisis general; en el apartado 6 se discute los resultados con otros estudios; en el aparatado 7 se presenta las principales conclusiones y en el apartado 8 se exponen algunas recomendaciones, así como también líneas futuras de trabajo.

\section{ANTECEDENTES}

A nivel del contexto mundial, en estudios desarrollados en España encontraron que el estudiantado maneja la información en un nivel de ejecución entre medio y elevado (Fajardo, Villalta y Salmerón, 2016). En otro estudio, las actividades que incorporan formaciones específicas sobre alfabetización informacional permite potenciar las habilidades informacionales, además de que existe diferencias entre sexo después de una intervención (Hernández, Vall y Boter, 2018). Nieto (2018), en un estudio comparativo encontró que en la población estudiantil, quienes están bajo una metodología, demuestran habilidades digitales significativamente mejores, especialmente en lo que respecta a la competencia digital comunicativa. Fernández y Manzano (2018) identificaron que el origen socioeconómico del estudiantado influye en el nivel de competencia digital a través del acceso a las TIC en el hogar. Respecto a la competencia digital de información, el estudiantado identifica ideas y se posicionan respecto a la información, pero tienen dificultades para interpretar información, localizar errores y calificar su fiabilidad (Valverde, Pro y Gonzáles, 2019). En otro estudio se encontró que la población estudiantil tiene una autopercepción bastante alta, especialmente en la dimensión de comunicación; pero en pruebas que evalúan dichas competencias, los resultados quedan algo por debajo de la autopercepción que se atribuían. Los autores mencionan que es necesario fortalecer la competencia digital de búsqueda y evaluación de la información (García, Martínez y Rodríguez, 2020). En cuanto a políticas sobre alfabetización en medios digitales de educación secundaria en España, no se han desarrollado convenientemente (Medina, Briones y Hernández, 2017). Así también, Sancho y Padilla (2016) mencionan que si bien existen políticas públicas de estado para desarrollar las competencias digitales y que estas se están integrando como competencias transversales en los currículos de estudios, no todo el personal docente utiliza las TIC regularmente. Canfarotta, Wolf y Casado (2018) encontraron que el estudiantado, especialmente los italianos, perciben un uso deficiente de la tecnología en el aula y que prevalece el uso de la enseñanza tradicional.

En Taiwán, Chen, Chen, Wang y Tai (2017), a través del método Fuzzy Delphi, determinaron seis dimensiones de competencias digitales, 21 subdimensiones y 81 competencias; las dimensiones son comunicación y colaboración, alfabetización en TIC, gestión de identidad, habilidades de aprendizaje, alfabetización informacional y alfabetización mediática. En Noruega, Hatlevik, Guðmundsdóttir y Loi (2015) identificaron que las condiciones en el hogar, el capital cultural, la integración lingüística, los logros 
académicos y la orientación al dominio predicen la competencia digital. En Polonia, Romaniuk (2015) encontró que las competencias digitales no son lo suficientemente altas y que no guardan relación con el número de dispositivos que poseen, el tiempo de uso ni con el tiempo de acceso diario al internet, además sugiere implementar un programa de formación en competencias digitales desde la educación primaria, para que en la secundaria demuestren habilidades básicas.

En Estados Unidos de Norte América, Biasini (2018) encontró que el mundo digital utilizado como tema de contenido desarrolla competencias y alfabetizaciones digitales en el estudiantado; asimismo, les permite reflexionar sobre el impacto de las TIC en su aprendizaje, en su vida personal en contextos sociales y profesionales. También, Pérez y Rodríguez (2015) concluyen que es necesario evaluar las competencias digitales en todos los niveles educativos para proponer políticas educativas, mejoras en los planes de estudios y programas de aprendizaje coherentes.

En el contexto latinoamericano, Henríquez, Gisbert y Fernández (2018) encontraron que la competencia mejor lograda es la de búsqueda y acceso a la información y la más baja está relacionada al aprendizaje social. En Ecuador, Marín, Rivera y Celly (2014) identificaron que alrededor del 18\% del estudiantado poseen alta competencia de formación audiovisual. Por otro lado, Navarrete y Mendieta (2018) señalan que hay avances respecto a usar las TIC en educación, pero también falta lograr la implementación de las tecnologías en todas las instituciones educativas. En Chile, Matamala (2015) identificó que el estudiantado presenta bajos niveles de competencias TIC, con diferencias según sexo, nivel socioeconómico, años de uso, frecuencia de uso y nivel de confianza en el uso de la computadora. En México, Vera de la O, Arias, Jiménez y Hernández (2018) identificaron que las habilidades se encuentran en el nivel de desarrollo deseable. Por otro lado, Contreras y Campa (2017) evidenciaron que el alumnado muestra tendencia de acceso y uso de internet para interacción y socialización, afirmación presentada porque el $84 \%$ usan redes sociales. En Colombia; Mantilla, Cedillo y Valenzuela (2014) encontraron que el estudiantado demuestra un nivel de competencias del $74 \%$. En el Perú, Paucar y Huamán (2015), en una provincia de la capital, identificaron que el perfil predominante dentro de la competencia informacional es el de recolector, es decir, el estudiantado no maneja la información de manera eficiente.

\section{REFERENTES CONCEPTUALES}

Las competencias digitales, según el Diario Oficial de la Unión Europea (2006), consiste en el

uso seguro y crítico de las tecnologías de la sociedad de la información (TSI) para el trabajo, el ocio y la comunicación. Se sustenta en las competencias básicas en materia de TIC: el uso de ordenadores para recuperar, evaluar, almacenar, producir, presentar e intercambiar información y comunicarse y participar en redes de colaboración a través de Internet. (p. 15)

Para el Instituto Nacional de Tecnologías Educativas y de Formación del Profesorado (2017) es usar las TIC de manera crítica, creativa y segura, para lograr objetivos en relación con el trabajo, empleo, aprendizaje, tiempo libre, inclusión y participación social. Las competencias informacionales son ciertas habilidades que el estudiantado debe adquirir para gestionar la información adecuadamente, según la CRUE-REVIUN (2013), citado por Bielva, Martínez, Herrera y Rodríguez (2015), estas habilidades son: buscar información, analizar y seleccionar información, organizar información, utilizar y comunicar la información de manera ética y legal. Para Hatlevik, Guðmundsdóttir y Loi (2015) son habilidades, conocimiento y actitudes que posee el estudiantado para utilizar los medios digitales en la participación, trabajo y resolución de problemas de manera autónoma y en colaboración, además de manera crítica, creativa y responsable; también identifican dimensiones como seguridad en internet, comunicación digital, recuperación de información digital, creación de contenido digital y resolución de problemas. Para Sancho y Padilla (2016) es evaluar la información de manera crítica y reflexiva; y contrastar y respetar las normas socialmente aceptadas para regular su uso y sus fuentes en diferentes medios. 
Por ende, las competencias digitales son un conjunto de capacidades que le permiten a la población estudiantil aprovechar los recursos de las TIC y utilizarlos en su proceso de aprendizaje de manera autónoma y permanente, competencias que les debe facilitar incorporarse favorablemente al mundo académico, profesional, laboral y personal. Asimismo, respecto a las competencias digitales, el INTEF (2017) los divide en cinco áreas y dentro de ellas se encuentran 21 competencias específicas. En cada una de estas competencias se especifican descriptores basados en términos de conocimientos, capacidades y actitudes, estas áreas competenciales son: Información y alfabetización informacional, comunicación y colaboración, creación de contenidos digitales, seguridad y resolución de problemas.

\section{Procedimientos metodológicos}

De una población total de 6109 estudiantes del nivel secundario de instituciones educativas públicas de la provincia de Tarma, región Junín, se extrajo una muestra de 665, de ellos $49.3 \%$ (328) son varones y 50.7\% (337) mujeres del tercero al quinto grado de educación secundaria. La selección de participantes fue a través de muestreo aleatorio estratificado, se utilizó como unidad de análisis la institución educativa más destacada de cada distrito de la provincia en mención. La muestra fue representativa con un nivel de confianza del $95 \%$.

$\mathrm{El}$ instrumento denominado Cuestionario de competencias digitales en estudiantes de educación secundaria se elaboró con base en INTEF (2017). Se tomó en consideración las 21 competencias dentro de las cinco áreas que lo componen:

Área de competencia 1. Información y alfabetización informacional: (1) Navegación, búsqueda y filtrado de información, datos y contenidos digitales. (2) Evaluación de información, datos y contenidos digitales. (3) Almacenamiento y recuperación de información, datos y contenidos digitales.

Área de competencia 2. Comunicación y colaboración: (1) Interacción mediante las tecnologías digitales. (2) Compartir información y contenidos digitales. (3) Participación ciudadana en línea. (4) Colaboración mediante canales digitales. (5) Netiqueta. (6) Gestión de la identidad digital.

Área de competencia 3. Creación de contenidos digitales: (1) Desarrollo de contenidos digitales. (2) Integración y reelaboración de contenidos digitales. (3) Derechos de autor y licencias. (4) Programación.

Área de competencia 4. Seguridad: (1) Protección de dispositivos. (2) Protección de datos personales e identidad digital. (3) Protección de la salud. (4) Protección del entorno.

Área de competencia 5. Resolución de problemas: (1) Resolución de problemas técnicos. (2) Identificación de necesidades y respuestas tecnológicas. (3) Innovación y uso de la tecnología digital de forma creativa. (4) Identificación de lagunas en la competencia digital.

La validez del instrumento, se organizó a través del Coeficiente de Validez de Contenido (CVC) desarrollado por Hernández (2011), obteniéndose 0.83. Respecto a la fiabilidad, la puntuación del Alfa de Cronbach del instrumento es de 0.95 . El cuestionario contiene preguntas relacionadas a las variables sexo y grado de educación, y a las competencias digitales del estudiantado; cada una tiene dos ítems, lo cual hace un total de 42 que conforman el instrumento, distribuidas en 21 competencias digitales dentro de las cinco áreas. La escala de medición del instrumento fue en base a los cuatro niveles de logro de la evaluación de la competencia: en inicio, en proceso, logro esperado y logro destacado (Ministerio de Educación, 2016).

Para la aplicación del instrumento se solicitó permiso a través de un documento formal a los directores de las instituciones educativas públicas seleccionadas. Después de la autorización, ya en los salones de clase se explicó al estudiantado el objetivo del estudio, el procedimiento del llenado del cuestionario y la confidencialidad de la respuesta; solo se aplicó el instrumento al estudiantado que accedieron participar voluntariamente. Los instrumentos se aplicaron desde el mes de junio hasta el mes de agosto del año 2019.

En cuanto al análisis de datos, las variables cualitativas se presentan según su distribución de frecuencias y porcentajes. Se han efectuado análisis no paramétricos después de comprobar el incumplimiento de los supuestos de normalidad, para analizar las diferencias de la variable sexo se llevó a cabo la prueba U de Mann- 
Whitney y para la variable grado se utilizó la prueba Kruskal-Wallis, porque presentan más de dos valores de respuesta.

\section{Resultados}

En la Tabla 1, se presenta los principales resultados vinculados al área de competencia digital de información y alfabetización informacional, dentro del cual se muestra los resultados de las tres competencias digitales que corresponden al área en mención.

TABLA 1

Área de competencia digital de información y alfabetización informacional

\begin{tabular}{|c|c|c|c|c|c|c|c|c|}
\hline \multirow[t]{2}{*}{ Competencia digital } & \multicolumn{2}{|c|}{ En inicio } & \multicolumn{2}{|c|}{ En proceso } & \multicolumn{2}{|c|}{$\begin{array}{l}\text { Logro } \\
\text { esperado }\end{array}$} & \multicolumn{2}{|c|}{ Logro destacado } \\
\hline & f & $\%$ & F & $\%$ & f & $\%$ & f & $\%$ \\
\hline 1. Navegación, búsqueda y filtrado de información, datos y contenidos digitales & 14 & $2.1 \%$ & 58 & $8.7 \%$ & 397 & $59.7 \%$ & 196 & $29.5 \%$ \\
\hline 2. Evaluación de información, datos y contenidos digitales & 16 & $2.4 \%$ & 122 & $18.3 \%$ & 403 & $60.6 \%$ & 124 & $18.6 \%$ \\
\hline 3. Almacenamiento y recuperación de información, datos y contenidos digitales & 42 & $6.3 \%$ & 131 & $19.7 \%$ & 386 & $58.0 \%$ & 106 & $15.9 \%$ \\
\hline Total & 8 & $1.2 \%$ & 121 & $18.2 \%$ & 466 & $70.1 \%$ & 70 & $10.5 \%$ \\
\hline
\end{tabular}

Fuente: Elaboración propia.

De la competencia digital 1, en el 59.7\% (397) de estudiantes prevalece el nivel de logro esperado, de ellos $27.8 \%$ (185) son varones y $31.9 \%$ (212) mujeres, con una diferencia significativa ( $U=50748.500$, $p=.036)$. En relación con el grado que cursa el estudiantado, también se encontró que predomina el nivel de logro esperado, donde los de $5^{\circ}$, en un $20.5 \%$ (136), son los que presentan mayor frecuencia, sin diferencia significativa $(\mathrm{H}=2.951, p=.229)$.

En cuanto a la competencia digital 2, en el 60.6\% (403) de estudiantes predomina el nivel de logro esperado, de ellos el 28.3\% (188) son varones y el 32.3\% (215) mujeres, sin diferencia significativa ( $U=52402.000$, $p=.186)$. En relación con el grado, también se identificó que prevalece el nivel de logro esperado, donde los de $5^{\circ}$, en un $21.8 \%$ (145), son los que presentan mayor frecuencia, sin diferencia significativa $(\mathrm{H}=3.976$, $p=.137)$.

De la competencia digital 3, en el 58.0\% (386) de estudiantes prepondera el nivel de logro esperado, de ellos 27.1\% (180) son varones y el 31.0\% (206) son mujeres, con diferencia significativa ( $U=49616.000$, $p=.010)$. En relación con el grado se identificó que prevalece el nivel de logro esperado, donde los de $5^{\circ}$, en un $21.1 \%$ (140), son los que presentan mayor frecuencia, sin diferencia significativa $(H=2.749, p=.253)$.

$\mathrm{Al}$ analizar a nivel del área, el 70.1\% (466) de estudiantes presentan un nivel de logro esperado de la competencia información y alfabetización informacional, es decir, evidencian un nivel esperado en cuanto al manejo satisfactorio de las competencias digitales que están dentro de esta área. De ellos 33.4\% (222) son varones y $36.7 \%$ (244) mujeres, no se muestra diferencia significativa $(\mathrm{U}=52816.500, p=.219)$; en cuanto al grado, también prevalece este nivel de logro, donde los de $5^{\circ}$, en un $25.0 \%$ (166), son los que presentan mayor frecuencia, sin diferencia significativa $(\mathrm{H}=5.274, p=.072)$.

A continuación, se muestra en la Tabla 2 los principales resultados del área de competencia digital de comunicación y colaboración, donde además se evidencia los resultados de las seis competencias que corresponden a esta área. 
TABLA 2

Área de competencia digital de comunicación y colaboración

\begin{tabular}{|c|c|c|c|c|c|c|c|c|}
\hline \multirow[t]{2}{*}{ Competencia digital } & \multicolumn{2}{|c|}{ En inicio } & \multicolumn{2}{|c|}{ En proceso } & \multicolumn{2}{|c|}{ Logro esperado } & \multicolumn{2}{|c|}{$\begin{array}{l}\text { Logro } \\
\text { destacado }\end{array}$} \\
\hline & $\mathbf{f}$ & $\%$ & f & $\%$ & $\mathbf{f}$ & $\%$ & f & $\%$ \\
\hline Interacción mediante las tecnologías digitales. & 40 & $60 \%$ & 165 & $24.8 \%$ & 337 & $50.7 \%$ & 123 & $18.5 \%$ \\
\hline Compartir información y contenidos digitales. & 88 & $13.2 \%$ & 207 & $31.1 \%$ & 301 & $45.3 \%$ & 69 & $10.4 \%$ \\
\hline Participación ciudadana en línea. & 151 & $22.7 \%$ & 274 & $41.2 \%$ & 197 & $29.6 \%$ & 43 & $6.5 \%$ \\
\hline Colaboración mediante canales digitales. & 133 & $20.0 \%$ & 205 & $30.8 \%$ & 265 & $39.8 \%$ & 62 & $9.3 \%$ \\
\hline Netiqueta & 31 & $4.7 \%$ & 118 & $17.7 \%$ & 394 & $59.2 \%$ & 122 & $18.3 \%$ \\
\hline 9. Gestión de la identidad digital. & 33 & $5.0 \%$ & 175 & $26.3 \%$ & 345 & $51.9 \%$ & 112 & $16.8 \%$ \\
\hline Total & 28 & $4.2 \%$ & 305 & $45.9 \%$ & 315 & $47.4 \%$ & 17 & $2.6 \%$ \\
\hline
\end{tabular}

Fuente: Elaboración propia.

De la competencia digital 4, en el 50.7\% (337) de estudiantes prevalece el nivel de logro esperado; de ellos, $25.4 \%$ (169) son varones y $25.3 \%$ (168) mujeres, sin diferencia significativa $(U=48358.500, p=.002)$. En cuanto al grado, también se encontró que predomina el nivel de logro esperado, donde los de $4^{\circ}(18.3 \%)$ y $5^{\circ}$ (18.3\%), son los que presentan mayor frecuencia, con diferencia significativa $(\mathrm{H}=26.150, p<.00)$.

En cuanto a la competencia digital 5 , en el 45.3\% (301) de estudiantes predomina el nivel de logro esperado, de ellos el 22.1\% (147) son varones y el 23.2\% (154) mujeres, con diferencia significativa (U= 49585.500, $p=.014)$. Del grado, también se identificó que prevalece el nivel de logro esperado, donde los de $4^{\circ}(15.3 \%)$ y $5^{\circ}(15.3 \%)$, son los que presentan mayor frecuencia, con diferencia significativa $(\mathrm{H}=10.44, p=.005)$.

En relación con la competencia digital 6, en el $41.2 \%$ (274) de estudiantes prepondera el nivel de logro en proceso, de ellos $20.0 \%$ (133) son varones y el 21.2\% (141) son mujeres, con diferencia significativa $(\mathrm{U}=$ $45669.500, p<.00)$. Del grado se identificó que prevalece el nivel de logro en proceso, donde los de $3^{\circ}$, en un $15.5 \%$ (103), son los que presentan mayor frecuencia, sin diferencia significativa $(\mathrm{H}=.511, p=.774)$.

En cuanto a la competencia digital 7, en el 39.8\% (265) de estudiantes prevalece el nivel de logro esperado, de ellos 19.8\% (132) son varones y 20.0\% (133) mujeres con diferencia significativa $(U=45724.000, p<.00)$. En relación con el grado se encontró que predomina el nivel de logro esperado, donde los de $4^{\circ}$, en un $14.6 \%$ (97), son los que presentan mayor frecuencia, no habiendo diferencia significativa $(\mathrm{H}=.893, p=.640)$.

De la competencia digital 8, en el 59.2\% (394) de estudiantes predomina el nivel de logro esperado, de ellos el 28.1\% (187) son varones y el 31.1\% (207) mujeres, sin diferencia significativa ( $U=53831.500, p=.511)$. En relación con el grado se identificó que prevalece el nivel de logro esperado, donde los de $5^{\circ}$, en un $20.9 \%$ (139), son los que presentan mayor frecuencia, sin diferencia significativa $(\mathrm{H}=3.986, p=.136)$.

En relación con la competencia digital 9, en el 51.9\% (345) de estudiantes prepondera el nivel de logro esperado, de ellos $25.1 \%$ (167) son varones y el 26.8\% (178) son mujeres, con diferencia significativa $(\mathrm{U}=$ $50006.500, p=.020)$. En relación con el grado se identificó que prevalece el nivel de logro esperado, donde los de $5^{\circ}$, en un $18.9 \%$ (126), son los que presentan mayor frecuencia, con diferencia significativa $(\mathrm{H}=6.163$, $p=.046)$.

Finalmente, el estudiantado presenta un nivel de logro esperado en las competencias digitales de interacción mediante las tecnologías digitales, compartir información y contenidos digitales, colaboración mediante canales digitales, netiqueta y gestión de la identidad digital; y un nivel de logro en proceso en la competencia digital de participación ciudadana en línea. Analizando a nivel del área, el $47.4 \%$ (315) del estudiantado presenta un nivel de logro esperado de la competencia de comunicación y colaboración, es decir, evidencian un nivel esperado en cuanto al manejo satisfactorio de las competencias digitales que están dentro de esta área. De ellos 24.2\% (161) son varones y 23.2\% (154) mujeres, con diferencia significativa (U= 
49572.500, $p=.010$ ); en cuanto al grado prevalece este nivel de logro, donde los de $5^{\circ}$, en un 18.0\% (120), son los que presentan mayor frecuencia, con diferencia significativa $(\mathrm{H}=11.166, p=.004)$.

A continuación, se presenta en la Tabla 3 los principales resultados del área de competencia digital de creación de contenidos digitales, dentro del cual se observa los resultados de las cuatro competencias digitales que corresponden al área en mención.

TABLA 3

Área de competencia digital creación de contenidos digitales

\begin{tabular}{|c|c|c|c|c|c|c|c|c|}
\hline \multirow[t]{2}{*}{ Competencia digital } & \multicolumn{2}{|c|}{ En inicio } & \multicolumn{2}{|c|}{ En proceso } & \multicolumn{2}{|c|}{ Logro esperado } & \multicolumn{2}{|c|}{$\begin{array}{l}\text { Logro } \\
\text { destacado }\end{array}$} \\
\hline & f & $\%$ & F & $\%$ & f & $\%$ & f & $\%$ \\
\hline 10. Desarrollo de contenidos digitales. & 34 & $5.1 \%$ & 150 & $22.6 \%$ & 361 & $54.3 \%$ & 120 & $18.0 \%$ \\
\hline 11. Integración y reelaboración de contenidos digitales. & 34 & $5.1 \%$ & 145 & $21.8 \%$ & 376 & $56.5 \%$ & 110 & $16.5 \%$ \\
\hline 12. Derechos de autor y licencias. & 44 & $6.6 \%$ & 318 & $47.8 \%$ & 246 & $37.0 \%$ & 57 & $8.6 \%$ \\
\hline 13. Programación. & 92 & $13.8 \%$ & 245 & $36.8 \%$ & 266 & $40.0 \%$ & 62 & $9.3 \%$ \\
\hline Total & 15 & $2.3 \%$ & 295 & $44.4 \%$ & 322 & $48.4 \%$ & 33 & $5.0 \%$ \\
\hline
\end{tabular}

Fuente: Elaboración propia.

En cuanto a la competencia digital 10, en el 54.3\% (361) de estudiantes prevalece el nivel de logro esperado, de ellos 26.8\% (178) son varones y 27.5\% (183) mujeres con una diferencia significativa (U= 50069.000, $p=.021)$. En relación con el grado se encontró que predomina el nivel de logro esperado, donde los de $5^{\circ}$, en un $18.6 \%$ (124), son los que presentan mayor frecuencia, sin diferencia significativa $(\mathrm{H}=1.305, p=.521)$.

De la competencia digital 11, en el 56.5\% (376) de estudiantes predomina el nivel de logro esperado, de ellos el 28.3\% (188) son varones y el 28.3\% (188) mujeres, sin diferencia significativa $(\mathrm{U}=51230.000, p=$ $.069)$. En relación con el grado se identificó que prevalece el nivel de logro esperado, donde los de $5^{\circ}$, en un $20.8 \%$ (138), son los que presentan mayor frecuencia, con una diferencia significativa $(\mathrm{H}=8.712, p=.013)$.

Con relación a la competencia digital 12, en el 47.8\% (318) de estudiantes prepondera el nivel de logro en proceso, de ellos $21.7 \%$ (144) son varones y el $26.2 \%$ (174) son mujeres, sin diferencia significativa $(\mathrm{U}=$ $51985.500, p=.148)$. En relación con el grado se identificó que prevalece el nivel de logro en proceso, donde los de $5^{\circ}$, en un $16.5 \%$ (110), son los que presentan mayor frecuencia, sin diferencia significativa $(\mathrm{H}=.081$, $p=.960)$.

En cuanto a la competencia digital 13, en el 40.0\% (266) de estudiantes prevalece el nivel de logro esperado. Según sexo, el 21.7\% (144) de varones presenta un nivel de logro esperado y el 20.6\% (137) de mujeres evidencia logro en proceso, con una diferencia significativa $(U=44702.000, p<.00)$. En relación con el grado se encontró que predomina el nivel de logro esperado, donde los de $5^{\circ}$, en un $14.6 \%$ (97), son los que presentan mayor frecuencia, no habiendo diferencia significativa $(\mathrm{H}=3.681, p=.159)$.

Finalmente, el alumnado presenta un nivel de logro esperado en las competencias digitales de desarrollo de contenidos digitales, integración y reelaboración de contenidos digitales y programación, y un nivel de logro en proceso en la competencia digital derechos de autor y licencias. Analizando a nivel del área el 48.4\% (322) de estudiantes presenta un nivel de logro esperado de la competencia creación de contenidos digitales, es decir, evidencian un nivel esperado en cuanto al manejo satisfactorio de las competencias digitales que están dentro de esta área. De ellos 23.6\% (157) son varones y 24.8\% (165) mujeres, sin diferencia significativa $(\mathrm{U}=$ $51528.500, p=.091)$; en cuanto al grado prevalece este nivel de logro, donde los de $5^{\circ}$, en un 18.6\% (124), son los que presentan mayor frecuencia, con una diferencia significativa $(\mathrm{H}=7.786, p=.020)$. 
En la Tabla 4 se muestra los principales resultados vinculados al área de competencia digital de seguridad, dentro del cual se observa los resultados de las cuatro competencias digitales que corresponden al área en mención.

TABLA 4

Área de competencia digital de seguridad

\begin{tabular}{|c|c|c|c|c|c|c|c|c|}
\hline \multirow[t]{2}{*}{ Competencia digital } & \multicolumn{2}{|c|}{ En inicio } & En proceso & \multicolumn{2}{|c|}{ Logro esperado } & \multicolumn{3}{|c|}{ Logro destacado } \\
\hline & f & $\%$ & f & $\%$ & f & $\%$ & f & $\%$ \\
\hline 14. Protección de dispositivos. & 46 & $6.9 \%$ & 6149 & $22.4 \%$ & 335 & $50.4 \%$ & 135 & $20.3 \%$ \\
\hline 15. Protección de datos personales e identidad digital. & 41 & $6.2 \%$ & $6 \quad 126$ & $18.9 \%$ & 361 & $54.3 \%$ & 137 & $20.6 \%$ \\
\hline 16. Protección de la salud. & 48 & $7.2 \%$ & 136 & $20.5 \%$ & 370 & $55.6 \%$ & 111 & $16.7 \%$ \\
\hline 17. Protección del entorno. & 63 & $9.5 \%$ & 210 & $31.6 \%$ & 320 & $48.1 \%$ & 72 & $10.8 \%$ \\
\hline Total & 18 & $2.7 \%$ & $6 \quad 196$ & $29.5 \%$ & 411 & $61.8 \%$ & 40 & $6.0 \%$ \\
\hline
\end{tabular}

Fuente: Elaboración propia.

En cuanto a la competencia digital 14, en el $50.4 \%$ (335) de estudiantes prevalece el nivel de logro esperado, de ellos 26.8\% (178) son varones y 23.6\% (157) mujeres con una diferencia significativa (U=47903.000, $p=.001)$. En relación con el grado se encontró que predomina el nivel de logro esperado, donde los de $5^{\circ}$, en un $17.4 \%$ (116), son los que presentan mayor frecuencia, con una diferencia significativa $(\mathrm{H}=12.765$, $p=.002)$.

De la competencia digital 15 , en el $54.3 \%$ (361) de estudiantes predomina el nivel de logro esperado, de ellos el 25.9\% (172) son varones y el 28.4\% (189) mujeres, sin diferencia significativa (U= 54460.500, $p=.720)$. En relación con el grado se identificó que prevalece el nivel de logro esperado, donde los de $5^{\circ}$, en un $18.8 \%$ (125), son los que presentan mayor frecuencia, con una diferencia significativa $(\mathrm{H}=7.622, p=.022)$.

En relación con la competencia digital 16, en el $55.6 \%$ (370) de estudiantes prepondera el nivel de logro esperado, de ellos $27.1 \%$ (180) son varones y el 28.6\% (190) son mujeres, con una diferencia significativa $(\mathrm{U}=49825.000, p=.015)$. En relación con el grado se identificó que prevalece el nivel de logro esperado, donde los de $5^{\circ}$, en un $19.2 \%$ (128), son los que presentan mayor frecuencia, sin diferencia significativa $(\mathrm{H}=$ $1.765, p=.414)$.

En cuanto a la competencia digital 17 , en el $48.1 \%$ (320) de estudiantes prevalece el nivel de logro esperado, de ellos $26.3 \%$ (175) son varones y el $21.8 \%$ (145) son mujeres, con una diferencia significativa( $\mathrm{U}=$ $46029.000, p=<.00)$. En relación con el grado se encontró que predomina el nivel de logro esperado, donde los de $5^{\circ}$, en un $16.2 \%$ (108), son los que presentan mayor frecuencia, sin diferencia significativa $(\mathrm{H}=.536$, $p=.765)$.

Analizando a nivel del área, el 61.8\% (411) de estudiantes presenta un nivel de logro esperado de la competencia seguridad, es decir, evidencian un nivel esperado en cuanto al manejo satisfactorio de las competencias digitales que están dentro de esta área. De ellos 30.1\% (200) son varones y $31.7 \%(211)$ mujeres, sin diferencia significativa $(\mathrm{U}=51196.000, p=.056)$; en cuanto al grado prevalece este nivel de logro, donde los de $5^{\circ}$, en un $22.9 \%$ (152), son los que presentan mayor frecuencia, con una diferencia significativa $(\mathrm{H}=$ 9.674, $p=.008)$.

A continuación, se observa en la Tabla 5 los principales resultados vinculados al área de competencia digital de resolución de problemas, dentro del cual se muestra los resultados de las cuatro competencias digitales que corresponden al área en mención. 
TABLA 5

Área de competencia digital resolución de problemas

\begin{tabular}{|c|c|c|c|c|c|c|c|c|}
\hline \multirow[t]{2}{*}{ Competencia digital } & \multicolumn{2}{|c|}{ En inicio } & En proceso & \multicolumn{3}{|c|}{ Logro esperado } & \multicolumn{2}{|c|}{ Logro destacado } \\
\hline & f & $\%$ & $\mathrm{f}$ & $\%$ & f & $\%$ & f & $\%$ \\
\hline 18. Resolución de problemas técnicos. & 48 & $7.2 \%$ & 230 & $34.6 \%$ & 299 & $45.0 \%$ & 88 & $13.2 \%$ \\
\hline 19. Identificación de necesidades y respuestas tecnológicas. & 81 & $12.2 \%$ & 235 & $35.3 \%$ & 286 & $43.0 \%$ & 63 & $9.5 \%$ \\
\hline 20. Innovación y uso de la tecnología digital de forma creativa. & 208 & $31.3 \%$ & 228 & $34.3 \%$ & 184 & $27.7 \%$ & 45 & $6.8 \%$ \\
\hline 21. Identificación de lagunas en la competencia digital. & 51 & $7.7 \%$ & 173 & $26.0 \%$ & 345 & $51.9 \%$ & 96 & $14.4 \%$ \\
\hline Total & 36 & $5.4 \%$ & 361 & $54.3 \%$ & 248 & $37.3 \%$ & 20 & $3.0 \%$ \\
\hline
\end{tabular}

Fuente: Elaboración propia.

De la competencia digital 19, en el 43.0\% (286) de estudiantes predomina el nivel de logro esperado, de ellos el 21.8\% (145) son varones y el 21.2\% (141) mujeres, con una diferencia significativa (U=48748.500, $p=.005)$. En relación con el grado se identificó que prevalece el nivel de logro esperado, donde los de $5^{\circ}$, en un $15.2 \%$ (101), son los que presentan mayor frecuencia, sin diferencia significativa $(H=1.303, p=.521)$.

En relación con la competencia digital 20, en el 34.3\% (228) de estudiantes prepondera el nivel de logro en proceso. Cuando se analizó por sexo el 11.6\% (116) de varones presenta un nivel de logro en proceso y el $19.7 \%$ (131) de mujeres evidencia competencia en inicio, con una diferencia significativa ( $U=44253.000$, $p<.00)$. En relación con el grado se identificó que prevalece el nivel de logro en proceso, donde los de $4^{\circ}$, en un $12.5 \%$ (83), son los que presentan mayor frecuencia, sin diferencia significativa $(\mathrm{H}=5.690, p=.058)$.

En cuanto a la competencia digital 21, en el 51.9\% (345) de estudiantes prevalece el nivel de logro esperado, de ellos $26.6 \%$ (177) son varones y el $25.3 \%$ (168) son mujeres, con una diferencia significativa $(\mathrm{U}=43386.500, p=<.00)$. En relación con el grado se encontró que predomina el nivel de logro esperado, donde los de $4^{\circ}$, en un $17.9 \%$ (119), son los que presentan mayor frecuencia, sin diferencia significativa $(\mathrm{H}=$ $3.779, p=.151)$.

Finalmente, el estudiantado muestra un nivel de logro esperado en las competencias digitales de: resolución de problemas técnicos, identificación de necesidades y respuestas tecnológicas e identificación de lagunas en la competencia digital; y un nivel de logro en proceso en la competencia digital innovación y uso de la tecnología digital de forma creativa. Analizando a nivel del área, el 54.3\% (361) de estudiantes presenta un nivel de logro en proceso de la competencia resolución de problemas, es decir, están próximo al nivel esperado en cuanto al manejo satisfactorio de las competencias digitales que están dentro de esta área. De ellos $22.7 \%$ (151) son varones y $31.6 \%$ (210) mujeres, con una diferencia significativa $(U=42171.500, p=<.00)$; en cuanto al grado prevalece este nivel de logro, donde los de $3^{\circ}$, en un $20.0 \%$ (133), son los que presentan mayor frecuencia, sin diferencia significativa $(\mathrm{H}=5.725, p=.057)$.

De las competencias digitales en general, en el 52.2\% (347) de estudiantes prevalece el nivel de logro esperado, de ellos $27.2 \%$ (181) son varones y el $25.0 \%$ (166) son mujeres, con una diferencia significativa $(\mathrm{U}=$ $49771.500, p=.012)$. En relación con el grado se encontró que predomina el nivel de logro esperado, donde los de $5^{\circ}$, en un $19.5 \%$ (130), son los que presentan mayor frecuencia, además se observa que el 18.0\% (120) de estudiantes del $3^{\circ}$ evidencian mayor frecuencia en el nivel de logro en proceso, comparando los grados existe diferencia significativa $(\mathrm{H}=15.483, p=<.00)$.

A continuación, en la Figura 1 se observa los resultados de las 21 competencias digitales del alumnado, donde se aprecia que prevalece el nivel de logro esperado: 


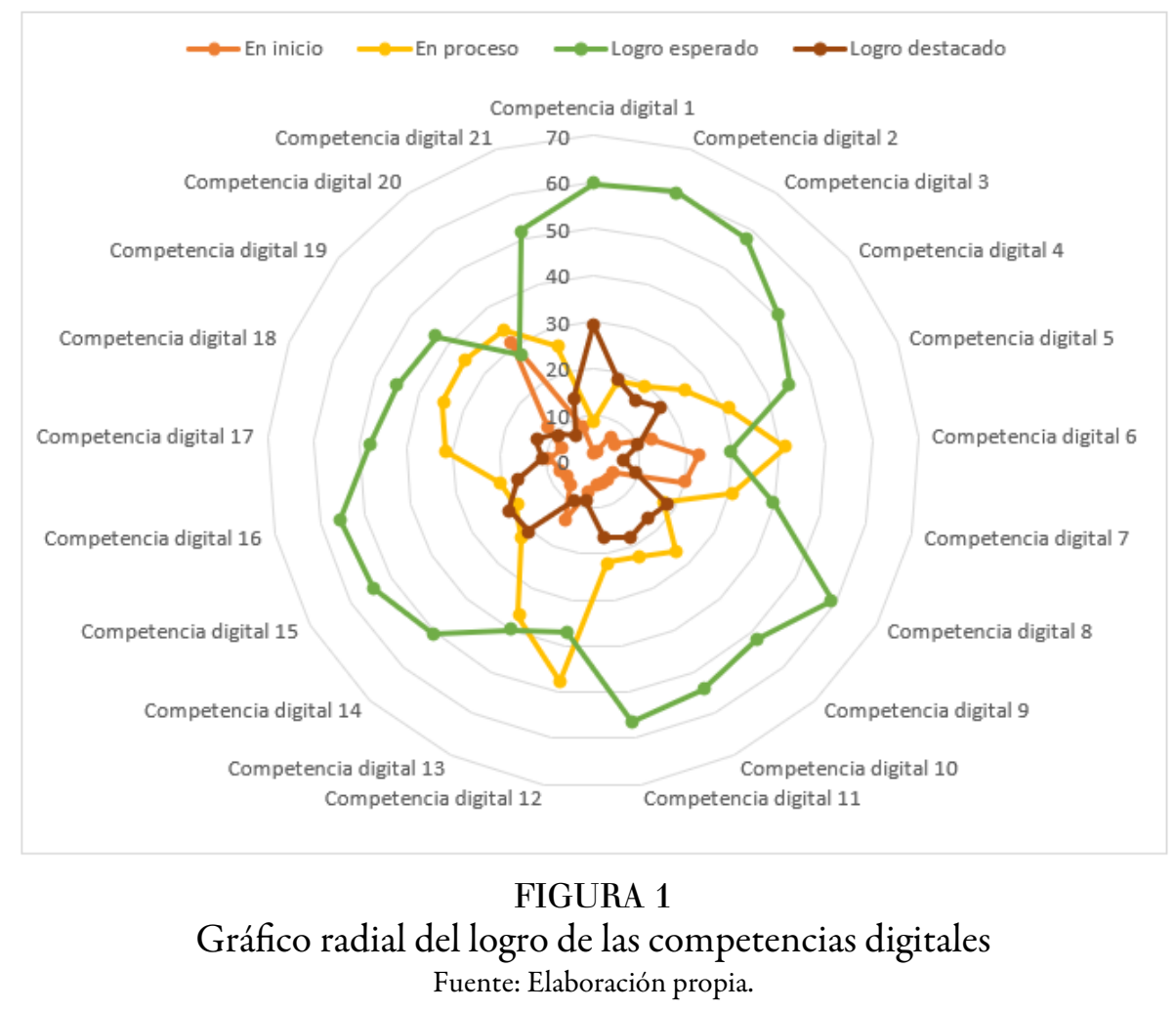

\section{Discusión}

La población estudiantil del nivel secundario en el contexto actual se encuentra inmersa en el uso de las TIC para desarrollar diversas actividades donde se incluyen las tareas escolares, es más, lo hacen con gran facilidad. En el Perú el 58.3\% del estudiantado del nivel secundario hacen uso de Internet (Instituto Nacional de Estadística e Informática [INEI], 2018) y va incrementándose cada año.

En el área de información y alfabetización informacional en las competencias digitales navegación, búsqueda y filtrado de información, datos y contenidos digitales; evaluación de información, datos y contenidos digitales; y almacenamiento y recuperación de información, datos y contenidos digitales; más del $58 \%$ de estudiantes evidencian un nivel de logro esperado, resultado que no concuerda con el estudio de ),Paucar y Huamán (2015 pero si coincide con los estudios de Fajardo, Villalta y Salmerón (2016); Henríquez, Gisbert y Fernández (2018) y Mantilla, Cedillo y Valenzuela (2014), también concuerda con el estudio de Valverde, Pro y Gonzales, (2019), en cuanto a búsqueda de información, más no en la evaluación de esta; tampoco coincide con los resultados de García, Martínez y Rodríguez (2020), quienes consideran que es necesaria una mayor formación, en especial en cuanto a búsqueda y evaluación de información. Los resultados del estudio dan a entender que el estudiantado navega, busca, filtra, evalúa, almacena y recupera información de internet de manera fácil y rápida, porque posiblemente desde edades más tempranas ya tenía acceso a internet y estaba familiarizado. También se observó que no todo el estudiantado ha logrado un nivel esperado de estás competencias, posiblemente por factores socioeconómicos, inadecuada infraestructura tecnológica en las instituciones educativas y limitadas competencias digitales de las y los docentes (Fernández y Manzano, 2018), lo cual implica que falta universalizar el desarrollo de esta área de competencia digital a nivel de toda la provincia, por lo que estudios posteriores deben orientarse a identificar el nivel de competencia digital de información y alfabetización informacional del personal docente; asimismo, la identificación de infraestructura tecnológica para, sobre ello, proponer alternativas de solución. 
En cuanto al área comunicación y colaboración, en las competencias digitales interacción mediante las tecnologías digitales, compartir información y contenidos digitales, colaboración mediante canales digitales, netiqueta y gestión de la identidad digital, más del $40 \%$ de estudiantes presentan nivel de logro esperado, mas no en la competencia digital participación ciudadana en línea (41.2\%), donde prevalece un nivel de logro en proceso, estos resultados concuerdan con Mantilla, Cedillo y Valenzuela (2014). En cuanto a la competencia digital interacción mediante las tecnologías digitales, concuerda con el estudio de Contreras y Campa (2017). Se puede deducir que el estudiantado se comunica por redes sociales, comparte información, elabora trabajos colaborativos, utiliza palabras y emoticones cuando se comunica y maneja su perfil social de manera satisfactoria porque está inmerso desde sus dispositivos tecnológicos. Pero en cuanto a participar en línea para efectuar algún trámite o debatir sobre temas sociales, políticos, económicos, ambientales, educativos, etc., el estudiantado requiere orientación. Esto quizá se deba a que no tienen la necesidad de ejecutar algún trámite o que las instituciones no han implementado. $Y$ en cuanto a participar en debates, posiblemente se deba a que no se sienten preparados para tal fin o en su defecto el profesorado no lo promueve. También es necesario destacar que estás competencias digitales se deben fortalecer a nivel de todo el alumnado con la finalidad de llegar a un nivel de logro destacado.

Referente al área creación de contenidos digitales, en las competencias digitales desarrollo de contenidos digitales, integración y reelaboración de contenidos digitales y programación se evidencia un logro esperado en más del 40\% de estudiantes, resultados concordantes con Mantilla, Cedillo y Valenzuela (2014). De la competencia digital derechos de autor y licencias (47.8\%), se observa un logro en proceso. Se observó que la población estudiantil conoce y utilizan herramientas tecnológicas para crear contenidos digitales, además utilizan información de la red para modificar y adecuar a sus tareas, también conocen la configuración de los dispositivos tecnológicos y además crean páginas web, aplicaciones entre otros de manera sencilla, esto posiblemente se deba a que el estudiantado está en interacción constante con contenidos digitales. Pero en cuanto a respetar derechos de autor y el manejo de licencias al usar la información, se observa que el estudiantado requiere acompañamiento u orientación para fortalecer esta competencia. También se requiere seguir trabajando para que estás competencias digitales se desarrollen a nivel de todo el estudiantado, por ello, estudios posteriores deben abarcar herramientas para desarrollar, integrar y reelaborar contenidos digitales, fortalecimiento del conocimiento de programación y, principalmente, el uso de licencias en el manejo de información virtual y derechos de autor en el uso de las TIC.

Dentro del área seguridad, más del $48 \%$ de estudiantes presentan un logro esperado en las competencias digitales de protección de dispositivos, protección de datos personales e identidad digital; protección de la salud y protección del entorno; y no se encontró estudios similares para su comparación y análisis. De estos resultados se notó que el estudiantado protege sus dispositivos a través de patrones de desbloqueo o contraseñas, además de los virus informáticos, protegen sus cuentas de redes sociales y utilizan estrategias para este fin, también conocen los riesgos derivados del uso de dispositivos tecnológicos que pueden dañar su salud, además de que se esfuerzan por utilizar la tecnología de manera adecuada para reducir el impacto ambiental. Una vez más, posiblemente esto se deba a la interacción con las tecnologías desde temprana edad y que por experiencia han ido desarrollando dichas competencias digitales. Pero es necesario hacer hincapié en que estás competencias del área de seguridad se deben seguir fortaleciendo, ya que, en el contexto de la región central del Perú, Orosco y Pomasunco (2020) encontraron que los principales riesgos a los que se exponen los adolescentes son: acceso a contenidos inapropiados (46.2\%), sexting (24.9\%), cyberbullying (20.9\%), grooming $(20.8 \%)$, sexcasting $(14.8 \%)$, sextorsión $(12.3 \%)$; y esta problemática se deriva por la falta de protección de los dispositivos tecnológicos y del manejo inadecuado de los datos personales e identidad digital.

En el área de resolución de problemas en las competencias digitales de resolución de problemas técnicos, identificación de necesidades y respuestas tecnológicas e identificación de lagunas en la competencia digital, más del $43 \%$ de estudiantes presentan un logro esperado. Pero en la competencia digital innovación y uso 
de la tecnología digital de forma creativa (34.4\%) solo llegan a un nivel de logro en proceso, estos resultados concuerdan con Mantilla, Cedillo y Valenzuela (2014). De estos resultados se deduce que el estudiantado sabe reconocer características técnicas y resolver problemas de sus dispositivos tecnológicos, además buscan medios digitales para solucionar sus tareas académicas o alguna duda y saben a qué sitio web acudir cuando requieran y están actualizados respecto a los dispositivos y plataformas tecnológicas. Pero en el caso de crear trabajos digitales originales y publicarlos en la web existen limitaciones, por lo que es necesario fortalecer esta competencia. Se observa también que estas competencias digitales no fueron desarrolladas por la mayoría del estudiantado, más aún en cuanto a la competencia de innovación y uso de la tecnología en forma creativa se evidencia un logro en proceso, por ello se considera que el cuerpo docente tiene una ardua tarea para encaminar al estudiantado ir del nivel de logro esperado al destacado.

Cuando se analizó a nivel general, se identificó que 52.2\% de estudiantes presentan un nivel de logro esperado según orden de prioridad de las siguientes competencias digitales: información y alfabetización informacional, seguridad, creación de contenidos digitales y comunicación y colaboración. Es en la competencia resolución de problemas donde predomina un nivel de logro en proceso; además, también se identificó que los varones presentan mayores niveles de competencia digital frente a las mujeres. Resultados que concuerdan en Colombia con Mantilla, Cedillo y Valenzuela (2014), caso contrario encontró Matamala (2015) en Chile donde señala que un 48\% del estudiantado muestra un bajo nivel de competencias TIC y que las mujeres presentan mayores niveles de competencias. Tampoco concuerda con Vera de la O, Arias, Jiménez y Hernández (2018) donde identificó un nivel deseable.

Finalmente, el alumnado se encuentra inmerso en el uso constante de las tecnologías, es por ello que ha desarrollado diversas competencias digitales y en su mayoría de manera autónoma, lo cual debe ser considerado por el profesorado y autoridades educativas para que tomen decisiones en mejora del proceso de enseñanza aprendizaje con base en la tecnología, ya que si el personal docente presenta competencias digitales deseables, permitirían que el estudiantado tenga mayor competencia digital, ello también implica que para seleccionar al cuerpo docente se deben incorporar la evaluación de la competencia tecnológica (Fernández y Manzano, 2018). Para fortalecer y desarrollar las competencias digitales del estudiantado es imprescindible que el personal docente esté capacitado metodológicamente para usar las TIC (Navarrete y Mendieta, 2018), para que a partir de ello se generen estrategias metodológicas, tanto dentro como fuera de las aulas y estas no deben limitarse a un contenido o asignatura en particular más, sino de manera transversal (Balladares, Avilés y Pérez, 2016). Ello da a entender que no basta implementar con equipos tecnológicos ni tampoco enseñar al personal docente a usar las TIC de manera instrumental (Peñaherrera, 2012), además "las nuevas políticas deben hacerse cargo no solo de la compra de equipos, sino de inversión en capacitación y formación, en recursos educativos innovadores y en la articulación sistémica con las políticas públicas en educación" (Oficina Regional de Educación para América Latina y el Caribe [OREALC], 2013, p. 20).

\section{Conclusiones}

- En el área de competencia digital de información y alfabetización informacional, el estudiantado presenta un logro esperado de las competencias de navegación, búsqueda y filtrado de información, datos y contenidos digitales; evaluación de información, datos y contenidos digitales; almacenamiento y recuperación de información, y datos y contenidos digitales.

- Del área de competencia digital de comunicación y colaboración, el alumnado evidencia un logro en proceso de la competencia participación ciudadana en línea y un logro esperado en las competencias de interacción mediante las tecnologías digitales, compartir información y contenidos digitales, colaboración mediante canales digitales, netiqueta y gestión de la identidad digital.

- En cuanto al área de competencia digital creación de contenidos digitales, prevalece un logro en proceso en la competencia de derechos de autor y licencias; y en las competencias desarrollo de 
contenidos digitales, integración y reelaboración de contenidos digitales, y programación prevalece el nivel de logro esperado.

- En relación con el área de competencia digital de seguridad, el alumnado denota prevalencia en el nivel de logro esperado en las competencias de protección de dispositivos, protección de datos personales e identidad digital, protección de la salud y protección del entorno.

- Del área de competencia digital resolución de problemas, en la competencia innovación y uso de la tecnología digital de forma creativa muestra logro en proceso, y en las competencias de resolución de problemas técnicos, identificación de necesidades y respuestas tecnológicas e identificación de lagunas en la competencia digital alcanzó un logro esperado.

- A nivel general, el estudiantado presenta un nivel de logro esperado en áreas de las competencias digitales de información y alfabetización informacional, seguridad y creación de contenidos digitales; de igual forma en comunicación y colaboración. En el área de la competencia resolución de problemas es donde predomina un nivel de logro en proceso.

\section{RECOMENDACIONES}

A razón de los resultados y la revisión de la literatura se observa que estudiantes del nivel secundario tienen competencias digitales, por lo que se recomienda integrar estas competencias en el proceso de aprendizaje aprovechando las TIC, tarea que debe asumir el profesorado; y por otro lado, las autoridades educativas y políticas deben implementar con infraestructura tecnológica que permita una mejor conectividad y acceso a los diversos recursos educativos digitales. Además, como se ha podido apreciar, el tema de competencias digitales en estudiantes de educación secundaria o media ha sido abordado en diversos contextos, por lo tanto, es menester seguir investigando el tema y como trabajos futuros se sugiere ahondar en el nivel de logro de estudiantado de educación secundaria en el sector norte y sur del territorio peruano, afín de levantar una línea de partida para plantear investigaciones que permitan desarrollar una propuesta metodológica contextualizada para el fortalecimiento de las competencias digitales en el profesorado, de tal manera que se aprovechen los recursos tecnológicos digitales en pro de la optimización del proceso educativo, ampliando el contexto pedagógico físico al contexto digital. Otros estudios también podrían abordar: formación de competencias digitales; observación directa de competencias digitales y factores externos e internos para el desarrollo de competencias digitales.

\section{ReFERENCIAs Bibliográficas}

Área, M. (2009). Introducción a la tecnología educativa: manual electrónico. España: Universidad de La Laguna. Recuperado de https://libros.metabiblioteca.org/handle/001/415

Balladares, J., Avilés, M. y Pérez, H. (2016). Del pensamiento complejo al pensamiento computacional: retos para la educación contemporánea. Sophia, (21), 143-159. doi https://doi.org/10.17163/soph.n21.2016.06

Biasini, R. (2018). The digital world as a topic: developing digital competences in the Italian language class En R. Biasini y A. Proudfoot (Eds), Using digital resources to enhance language learning - case studies in Italian (pp. 95-105). doi https://doi.org/10.14705/rpnet.2018.24.801

Bielva, M., Martínez, F., Herrera, M. y Rodríguez, M. (2015). Diseño de un instrumento de evaluación de competencias informacionales en educación secundaria obligatoria a través de la selección de indicadores clave. Education in the Knowledge Society, 16(3), 124-143. doi https://doi.org/10.14201/eks2015163124143

Calderón, F. J. (2019). Impacto de las nuevas tecnologías en la masificación de la educación. Revista Scientific, 4(Ed.esp.), 173-187. doi https://doi.org/10.29394/Scientific.issn.2542-2987.2019.4.E.10.173-187

Canfarotta, D., Wolf, J. y Casado, R. (2018). Digital competences and teaching of classical languages: students'perception in Italian and Spanish schools. Trabajo presentado en el Sixth International Conference on Technological 
Ecosystems for Enhancing Multiculturality, Salamanca, España. doi https://doi.org/10.1145/3284179.32842 16

Chen. F., Chen, J., Wang, J. y Tai, D. (2017). Using Fuzzy Delphi Method to Construct Digital Literacy Competences for Junior High School Students [Uso del método Fuzzy Delphi para construir competencias de alfabetización digital para estudiantes de secundaria]. International Journal of Information and Education Technology, 7(9), 686-689. doi https://doi.org/10.18178/ijiet.2017.7.9.954

Contreras, C. y Campa, R. (2017). Caracterización del perfil de los estudiantes de secundarias en el acceso y uso de internet a partir de las TIC. Edutec, Revista Electrónica de Tecnología Educativa, (61), 1-21. doi https://doi.or $\mathrm{g} / 10.21556 /$ edutec.2018.61

Cruz, E. (2019). Importancia del manejo de competencias tecnológicas en las prácticas docentes de la Universidad Nacional Experimental de la Seguridad (UNES). Revista Educación, 43(1). doi https://doi.org/10.15517/REV EDU.V43I1.27120

Cruz, M. A., Pozo, M. A., Aushay, H. R. y Arias, A. D. (2019). Las Tecnologías de la Información y de la Comunicación (TIC) como forma investigativa interdisciplinaria con un enfoque intercultural para el proceso de formación estudiantil. e-Ciencias de la Información, 9(1), 2-15. doi https://doi.org/10.15517/eci.v1i1.33052

Diario Oficial de la Unión Europea. (30 de diciembre de 2006). Recomendación del parlamento europeo y del consejo de 18 de diciembre de 2006 sobre las competencias clave para el aprendizaje permanente. Diario Oficial de la Unión Europea. Recuperado de https://eur-lex.europa.eu/LexUriServ/LexUriServ.do?uri=OJ:L:2006:394:001 0:0018:ES:PDF

Fajardo, I., Villalta, E. y Salmerón, L. (2016). ¿Son realmente tan buenos los nativos digitales? Relación entre las habilidades digitales y la lectura digital. Anales de psicología, 32(1), 89-97. doi https://doi.org/10.6018/analesp s.32.1.185571

Fernández, A. D. (2018). La competencia digital del alumnado de educación secundaria en el marco de un proyecto educativo TIC (1:1). Edutec, Revista Electrónica de Tecnología Educativa, (63), 60-72. Recuperado de https:// doi.org/10.21556/edutec.2018.63.1027

Fernández, M. y Manzano, D. (2018). Análisis de las diferencias en la competencia digital de los alumnos españoles. Papers. Revista de Sociología, 103(2), 175-198. doi https://doi.org/10.5565/rev/papers.2369

García, H., Martínez, F. y Rodríguez, M. (2020). Evaluación de la competencia informacional observada y autopercibida en estudiantes de educación secundaria obligatoria en una región española de alto rendimiento PISA. Revista Electrónica Recuperado de Educare, 24(1), 1-17. https://doi.org/10.15359/ree.24-1.2

Hatlevik, O., Guðmundsdóttir, G. y Loi, M. (2015). Examining factors predicting students' digital competence [Examinar los factores que predicen la competencia digital de los estudiantes]. Journal of Information Technology Education: Research, 14, 123-137. Recuperado de https://doi.org/10.28945/2126

Hernández, R. (2011). Instrumentos de recolección de datos en Ciencias Sociales y Ciencias Biomédicas. Venezuela: Universidad Los Andes - Mérida.

Hernández, C., Vall, A. y Boter, C. (2018). Formación, la clave para mejorar las competencias informacionales en esalud del alumnado de bachillerato. Gaceta Sanitaria, 32(1), 48-53. Recuperado de https://doi.org/10.1016/j. gaceta.2016.12.005

Henríquez, P., Gisbert, M. y Fernández, I. (2018). La evaluación de la competencia digital de los estudiantes: una revisión al caso latinoamericano. Chasqui. Revista Latinoamericana de Comunicación, (137), 91-110. Recuperado de https://revistachasqui.org/index.php/chasqui/article/download/3511/3013

Instituto Nacional de Estadística e Informática [INEI]. (2018). Población que accede a internet. Perú: Portal del Estado Peruano. Recuperado de http://m.inei.gob.pe/estadisticas/indice-tematico/population-access-to-internet/

Instituto Nacional de Tecnologías Educativas y de Formación del Profesorado. [INTEF].(2017). Marco Común de Competencia Digital Docente. España: Ministerio de Educación, Cultura y Deporte. Recuperado de https://ap rende.intef.es/mccdd

Levano, L., Sánchez, S., Guillén, P., Tello, S., Herrera, N. y Collantes, Z. (2019). Competencias digitales y educación. Propósitos y Representaciones, 7(2), 569-588. doi http://dx.doi.org/10.20511/pyr2019.v7n2.329 
Mantilla, M., Cedillo, M. y Valenzuela, J. (2014). Competencias en TIC, desarrolladas por estudiantes de secundaria, de acuerdo con los Estándares de la Sociedad Internacional para la Tecnología en la Educación. Congreso Iberoamericano de Ciencia, Tecnología, Innovación y Educación. Congreso llevado a cabo en Buenos Aires. Recuperado de https://www.oei.es/historico/congreso2014/memoriactei/17.pdf

Marín, I., Rivera, D. y Celly, S. (2014). Estudio sobre formación en competencia audiovisual de profesores y estudiantes en el sur de Ecuador. Cuadernos.info, (35), 119-131. Recuperado de https://doi.org/10.7764/cdi.35.628

Matamala, C. (2015). Factores predictivos de las competencias TIC en alumnos chilenos de secundaria. Revista Iberoamericana de Educación, 67(1), 121-136. Recuperado de https://rieoei.org/RIE/article/download/267/4 94

Mateus, J. C. y Suárez, C. (2017). La competencia TIC en el nuevo currículo peruano desde la perspectiva de la educación mediática. EDMETIC, 6(2), 129-147. Recuperado de https://doi.org/10.21071/edmetic.v6i2.6908

Medina, F., Briones, A. J. y Hernández, E. (2017). Educación en medios y competencia mediática en la educación secundaria en España. Revista ICONO14 Revista Cientifica De Comunicación Y Tecnologias Emergentes, 15(1), 42-65. Recuperado de https://doi.org/10.7195/ri14.v15i1.1001

Ministerio de Educación. (2016). Currículo nacional de la educación básica. Lima: Ministerio de Educación.

Muñoz, M. D. y Nicaragua, R. (2014). Un acercamiento a la brecha digital en Costa Rica desde el punto de vista del acceso, la conectividad y la alfabetización digital. E-Ciencias de la Información, 4(1), 1-29. Recuperado de 10.15517/ECI.V4I1.12866

Navarrete, G. y Mendieta, R. C. (2018). Las TIC y la educación ecuatoriana en tiempos de internet: breve análisis. Espirales, revista multidisciplinaria de educación, 2(15), 123-136. Recuperado de https://revistaespirales.com/i ndex.php/es/article/download/220/165

Nieto, E. (2018). Exploring CLIL contribution towards the acquisition of cross-curricular competences: a comparative study on digital competence development in CLIL [Explorando la contribución de CLIL hacia la adquisición de competencias transversales: un estudio comparativo sobre el desarrollo de competencias digitales en CLIL]. Revista de Lingüistica y Lenguas Aplicadas, 13(1), 75-85. doi https://doi.org/10.4995/rlyla.2018.9023

Oficina Regional de Educación para América Latina y el Caribe. (2013). Enfoques estratégicos sobres las TICS en educación en América Latina y el Caribe. Santiago: OREALC/UNESCO. Recuperado de http://www.unesco.o $\mathrm{rg} / \mathrm{new} /$ fileadmin/MULTIMEDIA/FIELD/Santiago/images/ticsesp.pdf

Orosco, J. R. y Pomasunco, R. (2020). Adolescentes frente a los riesgos en el uso de las TIC. Revista electrónica de investigación educativa, 22, e17, 1-13. doi https://doi.org/10.24320/redie.2020.22.e17.2298

Paucar, E. L. y Huamán, L. E. (2015). Perfiles de competencia informacional en los estudiantes del VII ciclo de educación básica regular de una institución educativa pública de la provincia de Barranca (Tesis de maestría). Pontificia Universidad Católica del Perú, Perú. Recuperado de http://tesis.pucp.edu.pe/repositorio/handle/20.500.1240 $4 / 7049$

Peñaherrera, M. (2012). Uso de TIC en escuelas públicas de ecuador: análisis, reflexiones y valoraciones. Edutec. Revista Electrónica de Tecnología Educativa, (40). a201. Recuperado de https://www.edutec.es/revista/index.php/edu tec-e/article/view/364

Pérez, M. (2017). Uso actual de las tecnologías de información y comunicación en la educación médica. Revista Médica Herediana, 28(4), 258-265. doi http://dx.doi.org/https://doi.org/10.20453/rmh.v28i4.3227

Pérez, A. y Rodríguez, J. (2015). Digital literacy and digital competences in the educational evaluation: USA and IEA contexts. 3a Conferencia internacional sobre ecosistemas tecnológicos para mejorar la multiculturalidad. Conferencia llevada a cabo en Portugal. Recuperado de https://doi.org/10.1145/2808580.2808633

Programa Aprende de la Junta de Castilla y León [PAJCL]. (2011). Las TIC en Educación. España: Consejería de Fomento de la Junta de Castilla y León. Recuperado de http://cralopedevega.centros.educa.jcyl.es/sitio/upload /MANUAL_TIC_EN_EDUCACION_JCYL.pdf

Romaniuk, M. I. (2015). Digital Competences of Maria Grzegorzewska Academy of Special Education Students Method and Results of a Survey. International Journal of Electronics and Telecommunications, 61(3), 267-272. doi https://doi.org/10.1515/eletel-2015-0035 
Jhon Richard Orosco Fabian, et al. Competencias digitales en estudiantes de Educación Secundaria d...

Sancho, J. M. y Padilla, P. (2016). Promoting digital competence in secondary education: are schools there? Insights from a case study. Journal of New Approaches in Educational Research, 5(1), 57-63. Recuperado de https://doi .org/10.7821/naer.2016.1.157

Sandí, J. C. y Sanz, C. V. (2020). Juegos serios para potenciar la adquisición de competencias digitales en la formación del profesorado. Revista Educación, 44(1), 1-18. doi https://doi.org/10.15517/revedu.v44i1.37228

Valverde, D., Pro, A. y González, J. (2019). La información científica en Internet vista por estudiantes de Educación Secundaria Obligatoria: Un estudio exploratorio de sus competencias digitales. Revista Eureka sobre Enseñanza y Divulgación de las Ciencias, 17(1), 1101-1 - 1101-18. Recuperado de https://doi.org/10.25267/Rev_Eureka _ensen_divulg_cienc.2020v17.i1.1101

Vera de la O, F. J., Arias, L., Jiménez, S. y Hernández, G. J. (2018). Habilidades digitales en la educación secundaria y su capacidad tecnológica instalada. Revista boletín REDIPE, 7(11), 88-102. Recuperado de Recuperado de http s://revista.redipe.org/index.php/1/article/download/630/587/ 